\title{
New binary stars discovered by lunar occultations. VI.*
}

\author{
A. Richichi ${ }^{1}$, G. Calamai ${ }^{2}$, and B. Stecklum ${ }^{3}$ \\ 1 European Southern Observatory, Karl-Schwarzschildstr. 2, 85748 Garching bei München, Germany \\ 2 Osservatorio Astrofisico di Arcetri, Largo E. Fermi, 5, 50125 Firenze, Italy \\ 3 Thüringer Landessternwarte Tautenburg, Sternwarte 5, 07778 Tautenburg, Germany
}

Received 12 September 2001 / Accepted 8 November 2001

\begin{abstract}
We present additional results from our ongoing lunar occultation program. Observations are presented here for a total of seventeen sources, for the majority of which one or two new companions are detected. The sample comprises mostly field stars, spanning a large range of spectral types. Two infrared objects without optical counterpart are also included. The range of projected separations is $00^{\prime \prime} 007$ to 0.162 . We report on the following binary stars: IRAS 16576-2116, IRAS 19495-1628, IRAS 18033-1955, CGCS 3964, SAO 94064, EI Tau, SAO 93950, SAO 79217, SAO 79285 and SAO 80075. We found HD 247924 and SAO 139322 to be triple systems. In the case of SAO 93950, the revised analysis of two occultation events has provided the true position angle and separation of this system. We also observed the young T Tauri star GG Tau A, where we detected a significant orbital motion with respect to the latest available measurements. In two cases, SAO 79285 and SAO 139322, a companion was already claimed on the basis of Hipparcos results, but our observations are not entirely consistent with these latter. Finally, our sample includes four stars known to be binary, namely SAO 186497, SAO 162183, SAO 96753 and SAO 161192, for which we did not detect a companion. Among these, our negative detection of the companion of SAO 162183 poses strong constraints on the parameters of this presumed binary.
\end{abstract}

Key words. astrometry - occultations - binaries: close - binaries: spectroscopic - binaries: visual stars: individual: GG Tau A

\section{Introduction}

This paper presents recent results on binary stars measured in the framework of an ongoing program of near infrared lunar occultations (LO). The sources which we investigate with the high angular resolution offered by LO include close binaries, both among fields stars and among young objects in star forming regions. The previous papers of this series provided a discussion of the merits and drawbacks of the LO technique, of the data analysis methods, and of the telescopes and instrumentation used for the observations. In particular, a summary and discussion of the aims and results of our LO program with respect to binary stars has been given in Richichi et al. (2000, hereafter Paper V), where references to previous papers in the series are also given. Thus, the present paper is intentionally very brief on such aspects.

\footnotetext{
Send offprint requests to: A. Richichi, e-mail: arichich@eso.org

* Based on observations collected at TIRGO (Gornergrat, Switzerland), and at Calar Alto (Spain). TIRGO is operated by CNR-CAISMI Arcetri, Italy. Calar Alto is operated by the German-Spanish Astronomical Center.
}

\section{Observations}

Table 1 lists the observational parameters of the observed sources, in the same format as Paper V. In summary, Cols. 1 through 3 list the source identification, the date of the event and the telescope used. In this latter column, the symbols identify the telescopes. These are: $\mathrm{T}$ for the $1.5 \mathrm{~m}$ TIRGO telescope equipped with the FIRT photometer, C1 and $\mathrm{C} 2$ for the Calar Alto $1.23 \mathrm{~m}$ and $2.2 \mathrm{~m}$ telescopes equipped with the FIRPO photometer, SA for the South African Astronomical Observatory $1.9 \mathrm{~m}$ telescope (SAAO) equipped with the Mk III photometer. In all these cases, broad band $K$ filters were used. The symbol W denotes observations carried out at the $2.3 \mathrm{~m}$ Wyoming Infrared telescope (WIRO) equipped with an InSb fast photometer; for these, a broad-band $L$ filter was used. The characteristics of the acquisition systems and filters, or references to them, are provided in Richichi et al. (1996a, 1997).

Column 4 lists the diaphragm aperture, while Cols. 5 and 6 list the sampling time of the lightcurves, and the integration time for each data point in the case of FIRT. Columns 7 and 8 list the total magnitude of the star in the $V$ and $K$ filters. The $V$ magnitudes are taken from the literature. The $K$ magnitudes are from our own 
Table 1. List of the occultation events and of the circumstances of their observation.

\begin{tabular}{|c|c|c|c|c|c|c|c|c|c|c|}
\hline (1) & $(2)$ & $(3)$ & (4) & (5) & (6) & (7) & (8) & (9) & (10) & (11) \\
\hline Source & Date & Tel. & $D$ & $\Delta t$ & $\tau$ & $V$ & $K$ & Sp. & Dist. & Notes \\
\hline & $\mathrm{UT}$ & & "I & $\mathrm{ms}$ & ms & mag & mag & & $\mathrm{pc}$ & \\
\hline IRAS 16576-2116 & 21-09-93 & SA & 12 & 2.0 & - & $: 15.1$ & $4.0^{a}$ & & & new detection \\
\hline IRAS 19495-1628 & $24-09-93$ & SA & 12 & 1.0 & - & & $4.8^{a}$ & & & new detection \\
\hline SAO 186497 & $20-07-94$ & $\mathrm{C} 2$ & 17 & 2.0 & - & 3.8 & $3.0^{b}$ & B2III: & $: 350$ & Algol-type, not detected \\
\hline IRAS 18033-1955 & $11-07-95$ & $\mathrm{~W}$ & 6 & 2.0 & - & & & & & new detection \\
\hline CGCS 3964 & $11-07-95$ & $\mathrm{~W}$ & 6 & 2.0 & - & $: 12.2$ & & S:... & & new detection \\
\hline SAO 94064 & $13-10-95$ & $\mathrm{~T}$ & 21 & 2.4 & 2.0 & 8.7 & $7.1^{a}$ & G5 & & new detection \\
\hline SAO 162183 & 04-06-96 & $\mathrm{C} 2$ & 14 & 0.8 & - & 8.5 & $4.3^{c}$ & M2III & $: 600$ & not detected \\
\hline SAO 96753 & $17-03-97$ & $\mathrm{C} 1$ & 17 & 2.0 & - & 8.5 & $5.0^{b}$ & K0 & 210 & miscatalogued as binary \\
\hline SAO 161192 & $14-08-97$ & $\mathrm{~T}$ & 28 & 4.9 & 4.5 & 9.6 & $7.7^{a}$ & K0 & 46 & wide visual binary \\
\hline EI Tau & $10-01-98$ & $\mathrm{~T}$ & 21 & 2.7 & 2.3 & $: 13.0$ & $3.9^{a}$ & Svar... & & new detection \\
\hline SAO 93950 & 05-11-98 & $\mathrm{T}$ & 28 & 2.4 & 2.0 & 5.0 & 2.2 & K2III & 60 & double event \\
\hline SAO 93950 & $30-12-98$ & $\mathrm{C} 1$ & 17 & 2.0 & - & 5.0 & 2.2 & K2III & 60 & double event \\
\hline HD 247924 & $19-01-00$ & $\mathrm{~T}$ & 14 & 5.4 & 5.0 & 9.0 & $4.5^{a}$ & K7 & & triple \\
\hline GG Tau A & $27-07-00$ & $\mathrm{~T}$ & 28 & 7.5 & 7.0 & $: 12.3$ & $: 8$ & K6V:e & & known binary \\
\hline SAO 79217 & 01-04-01 & $\mathrm{T}$ & 14 & 10.5 & 10.0 & 8.7 & $6.9^{a}$ & K0 & & new detection \\
\hline SAO 79285 & 01-04-01 & $\mathrm{T}$ & 14 & 10.5 & 10.0 & 7.8 & $5.6^{a}$ & G5 & 270 & triple? \\
\hline SAO 80075 & 02-04-01 & $\mathrm{T}$ & 21 & 5.4 & 5.0 & 8.6 & $5.4^{a}$ & M0 & $>560$ & new detection \\
\hline SAO 139322 & 01-06-01 & $\mathrm{T}$ & 14 & 5.0 & 5.4 & 6.8 & $4.8^{a}$ & K0 & 119 & triple \\
\hline
\end{tabular}

${ }^{a}$ Estimated from the signal level during the LO event. ${ }^{b}$ Obtained from own photometry at the TIRGO telescope. ${ }^{c}$ Estimated from assumed $(V-K)$ for the given spectral type.

photometric data when available, or are otherwise estimated as indicated. A colon sign indicates known variability. In Cols. 9 and 10 we report the spectral types and distances, again extracted when available from the literature; in the case of multiple determinations, the most frequent or most recent was used. The distances are based on Hipparcos parallaxes, when available. Distance values preceded by a colon are our own estimates. These latter were obtained from the magnitude and spectral type, and using the appropriate luminosity class. These distance estimates are very approximate, and should be taken only as an order-of-magnitude indication. Finally, the last column reports a short comment on the nature of the detection. Table 2 lists the cross-identifications of the observed sources.

\section{Stars with positive binary detection}

The stars for which we could positively detect a companion are listed in Table 3, where the entries follow the same style as in Paper V. In particular, the columns list the absolute value of the fitted linear rate of the event $V$ in $\mathrm{m} / \mathrm{s}$, its deviation from the predicted rate $V_{\mathrm{t}}$ as computed by us, the local lunar limb slope $\psi$, the true position and contact angles, the signal-to-noise ratio (SNR), the projected separation, and the brightness ratio for each pair of components. When the SNR of the lightcurve was not sufficient to determine reliably the actual rate of the event,
Table 2. Cross identifications.

\begin{tabular}{|c|c|c|}
\hline IRAS 16576-2116 & V1203 Oph & Plaut 2- 80 \\
\hline IRAS 19495-1628 & $19^{\mathrm{h}} 52^{\mathrm{m}} 24^{\mathrm{s}} .6$ & $-16^{\circ} 20^{\prime} 28^{\prime \prime}(2000.0)$ \\
\hline SAO 186497 & HD 166937 & $\mu \operatorname{Sgr}$ \\
\hline IRAS 18033-1955 & $18^{\mathrm{h}} 06^{\mathrm{m}} 20.1$ & \\
\hline CGCS 3964 & Case 613 & $\begin{array}{r}-19^{\circ} 54^{\prime} 47^{\prime \prime}(2000.0) \\
\mathrm{C}^{*} 2544\end{array}$ \\
\hline SAO 94064 & HD 29601 & $\mathrm{AG}+18360$ \\
\hline SAO 162183 & HD 177581 & BD-18 5201 \\
\hline SAO 96753 & IDS $07128+1646 \mathrm{AB}$ & $\mathrm{AG}+16756$ \\
\hline SAO 161192 & IDS 18071-1915 A & BD-19 4901 \\
\hline EI Tau & $\mathrm{S} 1 * 116$ & $\mathrm{CSI}+17-05440$ \\
\hline SAO 93950 & $\mathrm{IRC}+20079$ & HD 28292 \\
\hline HD 247924 & $\mathrm{AG}+19491$ & $\mathrm{BD}+191082$ \\
\hline GG Tau A & IRAS $04296+1725$ & HBC 54 \\
\hline SAO 79217 & $\mathrm{BD}+221621$ & $\mathrm{AG}+22847$ \\
\hline SAO 79285 & HD 56790 & $\mathrm{AG}+22859$ \\
\hline SAO 80075 & $\mathrm{BD}+211805$ & $\mathrm{AG}+21920$ \\
\hline SAO 139322 & HD 116545 & BD-03 3462 \\
\hline
\end{tabular}

we list in Col. 2 the predicted one instead, in parentheses. In these cases, the limb slope is undetermined and we list the predicted values of $\mathrm{CA}$ and PA, also in parentheses. A more detailed discussion of the quantities in Table 3 is given in Richichi et al. (1996a).

In the following, for each of the stars with a positive binary (or multiple) detection we discuss our result in the context of available data. 
Table 3. Summary of binary detection results.

\begin{tabular}{|c|c|c|c|c|c|c|c|c|}
\hline (1) & $(2)$ & (3) & (4) & (5) & (6) & (7) & (8) & (9) \\
\hline Source & $|V|$ & $V / V_{\mathrm{t}}-1$ & $\psi$ & PA & $\mathrm{CA}$ & SNR & Sep. (mas) & Br. Ratio \\
\hline IRAS 16576-2116 & 0.8762 & $3 \%$ & 13 & 263 & 0 & 47.4 & $50.5 \pm 0.4$ & $11.0 \pm 0.3$ \\
\hline IRAS 19495-1628 & 0.8607 & $-1 \%$ & -2 & 84 & 16 & 37.6 & $20.5 \pm 1.3$ & $20.1 \pm 1.7$ \\
\hline IRAS 18033-1955 & 0.6919 & $-1 \%$ & -2 & 244 & -22 & 33.2 & $37.0 \pm 0.5$ & $19.6 \pm 0.7$ \\
\hline CGCS 3964 & 1.0020 & $24 \%$ & 6 & 272 & 12 & 97.2 & $20.2 \pm 0.2$ & $13.1 \pm 0.2$ \\
\hline SAO 94064 & $(0.5690)$ & & & $(128)$ & $(218)$ & 2.9 & $29.3 \pm 4.9$ & $5.3 \pm 1.0$ \\
\hline EI Tau & 0.9373 & $4 \%$ & 4 & 82 & 0 & 52.2 & $7.3 \pm 0.6$ & $14.6 \pm 1.1$ \\
\hline SAO 93950 & 0.7174 & $39 \%$ & -21 & 105 & 211 & 97.8 & $7.2 \pm 2.0$ & $28.1 \pm 6.5$ \\
\hline SAO 93950 & 0.8042 & $2 \%$ & 3 & 55 & -15 & 34.2 & $25.5 \pm 2.5$ & $79.4 \pm 19.2$ \\
\hline GG Tau A & $(0.6549)$ & & & $(301)$ & $(230)$ & 6.8 & $162.5 \pm 1.5$ & $1.4 \pm 0.1$ \\
\hline SAO 79217 & 0.4165 & $-10 \%$ & -4 & 152 & 52 & 12.7 & $17.0 \pm 2.4$ & $7.1 \pm 0.8$ \\
\hline SAO 79285 & 0.5488 & $-10 \%$ & -5 & 147 & 47 & 32.0 & $36.7 \pm 1.7$ & $11.0 \pm 0.6$ \\
\hline SAO 80075 & 0.5405 & $-13 \%$ & -8 & 57 & -48 & 24.7 & $17.0 \pm 1.0$ & $8.6 \pm 0.5$ \\
\hline \multicolumn{9}{|c|}{ triple systems } \\
\hline HD 247924 A-B & 0.8314 & $-2 \%$ & -8 & 77 & -11 & 44.5 & $9.1 \pm 0.5$ & $7.3 \pm 0.3$ \\
\hline HD 247924 A-C & & & & 257 & & 44.2 & $12.2 \pm 0.5$ & $7.6 \pm 0.3$ \\
\hline SAO 139322 A-B & 0.7419 & $-8 \%$ & -14 & 273 & -27 & 45.9 & $34.7 \pm 0.6$ & $8.5 \pm 0.2$ \\
\hline SAO 139322 A-C & & & & 93 & & 45.1 & $16.6 \pm 0.7$ & $10.2 \pm 0.4$ \\
\hline
\end{tabular}

\subsection{El Tau}

A brief account of the LO event for this star has been given in Richichi \& Calamai (2001), where we have reported on the measurement of the angular diameter of this S-type giant star. Additionally, in the present paper we report the new detection of a relatively faint and nearby companion. No high angular resolution measurement of this source were reported before in the literature.

\section{2. $S A O 93950$}

We have recorded two LO events for this star, which have revealed a companion and have permitted us to measure the angular diameter of the primary. These results have been reported already in Richichi et al. (1999) and Richichi \& Calamai (2001). The reason for which we include this star again in the present paper is that, contrary to what was reported in Richichi et al. (1999) where the companion was identified only in one of the two LO records, we have been able to detect the companion also in the other available trace. This was the result of a detailed analysis of the LO data, that we performed in order to obtain an unbiased diameter estimate (Richichi et al. 2001).

The two occultations, which were recorded only one month apart, make it now possible to derive the true separation and position angle of the system. We compute these values to be $28.2 \pm 3.2 \mathrm{mas}$ and $30^{\circ} \pm 5^{\circ}$ respectively, under the assumption that orbital motion between the two LO measurements can be neglected (see below). The brightness ratio of the companion is not in very good agreement between the two independent LO lightcurves, due to its faintness, but the large errors can explain this at least in part. The magnitude difference of the two components seems to be in the range $\Delta K=3.2$ to 4.8 .

Using the distance of $60 \mathrm{pc}$ as determined by Hipparcos, the semi-major axis of the system should then be $\geq 1.6$ AU. This leads to a period which could be of the order of about 2 years, assuming a system mass of $\approx 1 M_{\odot}$. Therefore, a difference between the two LO events of one month is small, but not negligible. It is likely that an orbital motion of $\gtrsim 10^{\circ}$ might have occurred between the two LO events, and the formal errors on position angle and separation quoted above should be considered as a lower estimate only. At the same time, this indicates the possibility of a detectable motion over a period of few years. A number of measurements by LO and speckle interferometry, all with negative results, are listed in Richichi et al. (1999). The only other positive measurement of the companion is that of Fekel et al. (1980), who reported a projected angular separation of 24 mas along $\mathrm{PA}=238^{\circ}$. The value of the separation is consistent with our determination, as well as the apparent reversal in direction of the position angle over the 19.7 years between our measurements and that of the authors above. The color of the companion, determined from the visual and near-IR brightness ratios, indicates a spectral type bluer than that of the primary.

\subsection{HD 247924}

By means of a set of LO data with good SNR, we have discovered two nearby companions to HD 247924. Only 
one previous measurement of this late-type star was reported in the literature, namely by Blow et al. (1982) who observed a LO event in the visible but did not report any deviation from a point source. Their negative finding can be explained, when one considers the rather faint magnitude of this star in the visible and the small separation and brightness of the two companions. We note that their brightnesses are similar (at least in the near infrared), which might be an indication of similar masses. The fact that their distances from the primary are similar but in opposite directions is consistent from this point of view, when one considers the stability of such a triple system.

\subsection{GG Tau $A$}

GG Tau is one of the most studied low-mass young stellar objects. Located in the Taurus star forming regions, at about $140 \mathrm{pc}$, it consists of two close binary systems (Leinert et al. 1993). The southern one, with a separation of $\approx 1^{\prime \prime}$. 4 , was too faint and too close to the border of the diaphragm to be detected reliably in our LO data. We detected instead the northern pair, GG Tau A+a. This latter is surrounded by a disk, which is detected in emission at millimeter wavelengths (Guilloteau et al. 1999) as well as in scattered light at shorter wavelengths (Roddier et al. 1996). The SNR of our data was not sufficient to detect the disk, and in the following we discuss only GG Tau A and its companion.

A LO observation was reported also by Simon et al. (2000), who measured it with a relatively slow sampling frequency. They were not able to measure directly the lunar limb rate, but they stated that using the predicted value in their analysis yielded a projected separation consistent with the value determined by Roddier et al. (1996) about 1.3 years earlier.

Also in our case the actual rate of motion of the lunar limb could not be measured reliably: although the sampling was quite sufficient, the small telescope size did not provide sufficient SNR. Using the predicted value, we obtain a projected separation of $0.1625 \pm 0$ ". 0015 along $\mathrm{PA}=301^{\circ} \pm 1^{\circ}$. This indicates a significant orbital motion from the latest available measurements (see Roddier et al. 1996). Unfortunately the qualitative orbital analysis given in this latter reference is not sufficient, given the measurements available at that time, to extrapolate an accurate position to compare with our LO measurements. On the other hand, our measurement should constrain significantly such orbital studies.

For what concerns the relative brightness of components, we note that although we lack a global $K$-band photometric measurement at the date of the event, our LO data indicate a magnitude difference of $0.37 \pm$ $0.05 \mathrm{mag}$, which seems to indicate a significant variation with respect to the determination of Simon et al. (2000). It should be noted that photometric variability is typical among young stellar objects (Kaas 1999).

\subsection{SAO 79285}

This star has been discovered to be a subarcsecond binary by the Hipparcos survey (HIP 35481), with separation and position angle of $00^{\prime \prime} 690$ and $70^{\circ}$, respectively. Fabricius \& Makarov (2000) have published photometry of both components, resulting in $\Delta B=1.65 \pm 0.09 \mathrm{mag}$ and $\Delta V=2.92 \pm 0.10 \mathrm{mag}$ (in the Tycho system).

The comparison of the Hipparcos results with that obtained from our LO data shows however some discrepancies. For example, the magnitude difference in the $K$ band has been determined by us to be $2.60 \pm 0.06 \mathrm{mag}$, which in order to be reconciled with those at shorter wavelengths would require a peculiar spectrum at least in one of the two components. More importantly, considering the projection of the true (Hipparcos) separation along the PA of the LO event, one would expect a separation of $\approx 0$ ". 155 , which is in strong contrast with our determination of $0 . \prime 037$. The Hipparcos parallax places this system at $270 \mathrm{pc}$, with a lower limit of $127 \mathrm{pc}$. Therefore, the expected orbital period should be $\gtrsim 10^{3}$ years, and effects of orbital motion between the epoch of the Hipparcos measurements and of our LO event should be negligible.

One possible explanation could be that the component detected by LO be in fact a third component in the system. This hypothesis would need to be confirmed by further observations. For the time being, we note that we have examined our LO lightcurve in a range of \pm 0 '. 23 from the expected time of occultation of the Hipparcos secondary. We can place an upper limit of $\Delta K=3.7$ with respect to the primary, on the presence of a companion with the Hipparcos parameters. We also note that in the case of SAO 139322, discussed below, what we find to be a triple star was reported as a binary in the analysis of the Hipparcos data.

\section{6. $S A O 139322$}

Similarly to SAO 79285, also SAO 139322 was previously detected by Hipparcos to be a binary star. Also in this case, the parameters of the binary companion and its differential colors (Fabricius \& Makarov 2000) are in disagreement with either one of the two companions that we find. For example, the projection of the true (Hipparcos) values of separation and position angle, $0^{\prime \prime} .400$ and $239^{\circ}$ respectively, along the position angle of our LO event would indicate an expected projected separation of the companion of $\approx 0^{\prime \prime} 33$, in contrast with the separations of our two detected companions of $00^{\prime \prime} 035$ and $00^{\prime \prime} 017$. As in the case of SAO 79285, orbital motions between the epoch of the Hipparcos parallax and that of our measurement should be negligible.

The result derived from our LO data can be considered convincing (see Fig. 1). In order to reconcile this finding with the result derived from the Hipparcos data, we tentatively suggest that having forced a binary solution in what probably is a triple system might have led to some biased estimates. The analysis of the Hipparcos data for 


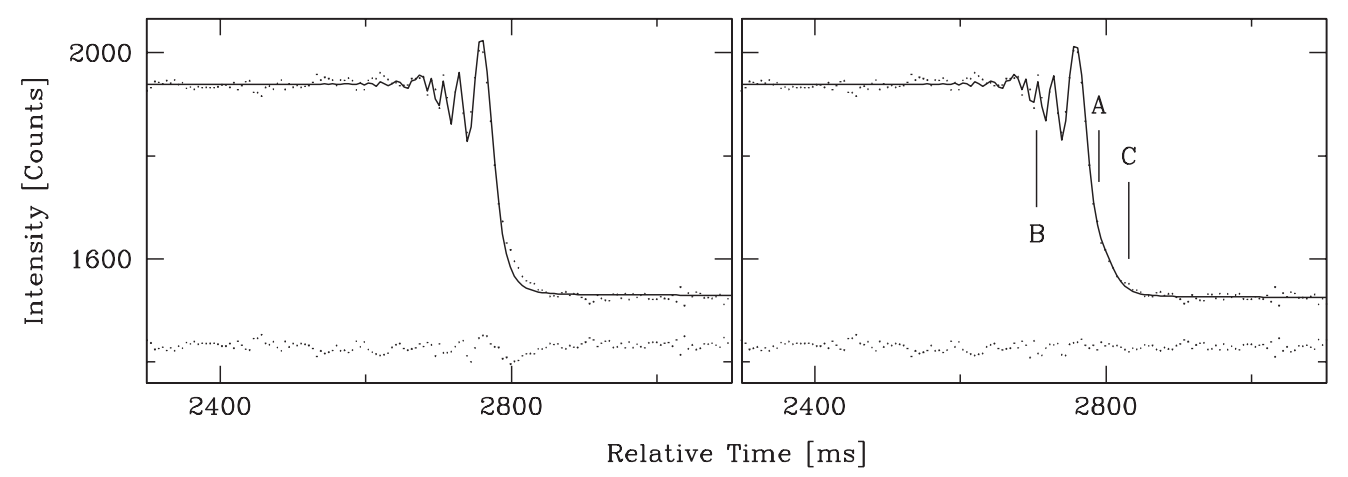

Fig. 1. Example of a set of lunar occultation data, illustrating the detection of SAO 139322 as a triple star. The left panel shows the least squares best fit (solid line) to the occultation data (dots), by a binary star model. The trace at the bottom are the fit residuals, shifted by an arbitrary offset. The panel on the right shows the result for a triple star model. In the right panel, the time of occultation of each component has been marked.

this system using a triple star solution might help to clarify the situation.

\subsection{Remaining stars}

There is almost no literature available for IRAS 165762116, IRAS 19495-1628, IRAS 18033-1955, CGCS 3964, SAO 94064, SAO 79217 and SAO 80075. For all these stars, our measurements by LO have identified for the first time a nearby companion, with the parameters listed in Table 3 .

IRAS 16576-2116 is identified as a faint Mira-type variable. Optical images show a nearby star at about $8^{\prime \prime}$ along $\mathrm{PA} \approx 40^{\circ}$, but this is much further away than our detected companion. The other two IRAS sources in our list have no optical identification. IRAS 19495-1628 has two nearby relatively bright stars, which however were not included in our LO observations. IRAS 18033-1955 has a nearby $\left(\approx 5^{\prime \prime}\right)$ source which is barely noticeable in visible POSS plates, but is quite brighter in red and infrared plates, and which could represent the optical counterpart.

CGCS 3964 is a relatively poorly studied carbon star. It was first listed as such by Nassau \& Blanco (1957), although with a large positional error. Subsequent works have refined position and provided some magnitudes. The latest work including this star is that of Skiff (1999), which mentions magnitudes $B=18.4$ and $I=8.1$.

SAO 94064 was listed in a previous compilation of LO events observed from TIRGO (Richichi et al. 1996b), without having been recognized as a binary at that time. SAO 80075 was reported in one of the first papers on LO results by Evans (1971), in which the author remarked that the angular diameter of this star might be detectable but did not attempt a detailed analysis of the data. In any case, no mention of duplicity was made.

\section{Stars with negative binary detection}

As in previous papers of this series, we list in Table 4 a few stars which are known to be binary but for which we could not detect the companion in our LO observation. The table lists also some basic parameters regarding the geometry and quality of the LO event, and the possible causes for non detection.

SAO 186497 is a bright Algol-type star for which a good SNR LO event could be recorded. The Hipparcos determination of the parallax to this star is too small to be reliable, but in any case indicates a distance of several hundreds pc. We derive a spectroscopic parallax of $\approx 350$ pc. Given the large distance, there is no doubt that the angular separation of the companion is beyond possibility of direct detection by high angular resolution methods. We note that negative results by speckle interferometry at visual wavelengths were recorded also by Bonneau et al. (1980), by Hartkopf \& McAlister (1984), Isobe et al. (1992) and Miura et al. (1992). SAO 186497 also has several other wide companions, which however are too faint and/or too distant to be detected in our LO light curve.

SAO 162183 is included in the list of double stars maintained by the International Occultation Timing Association (H. Bulder, private communication). This star was reported as binary in a visual non-grazing occultation, with two components of equal brightness separated by $0 !^{\prime} 1$ along $\mathrm{PA}=94^{\circ}$. Our measurement, obtained with good SNR, was recorded along a position angle very similar to that of the detection event, though in opposite direction. Hence, it should have easily revealed the two components. The possibility that the two stars could have different colors is hard to defend: considering our detection threshold of over $4 \mathrm{mag}$ in the near-IR and the magnitude of the star, the companion would have to be a late B star or bluer, whose spectral signature would have probably been already noticed. Regarding the possibility of a very relevant orbital motion between the original epoch of detection (1977) and our measurement, we notice that a spectroscopic parallax estimate puts the distance at $\gtrsim 500 \mathrm{pc}$. This is also consistent with the absence of a parallax determination by Hipparcos, although the star was observed by this satellite. The resulting semi-axis of the orbit would then be $\geq 25 \mathrm{AU}$, implying an orbital period in excess of 
Table 4. Summary of negative detection results.

\begin{tabular}{ccccl}
\hline \hline$(1)$ & $(2)$ & $(3)$ & $(4)$ & $(5)$ \\
Source & $\psi$ & PA & SNR & Notes \\
\hline SAO 186497 & 3 & 129 & 80.4 & Insufficient resolution \\
SAO 162183 & 12 & 278 & 58.8 & Visual detection \\
SAO 96753 & 10 & 147 & 5.3 & Not binary \\
SAO 161192 & 0 & 101 & 3.0 & Wide binary \\
\hline \hline
\end{tabular}

100 years. We have to conclude that our negative detection is hard to reconcile with a double star with the above mentioned parameters.

SAO 96753 is included in our list of negative detections, since this star was originally listed as double (WEI 15AB). However, some discrepancies appeared when we tried to find more details in the available references. Following our inquiries, the staff at the Centre de Données Astronomiques (CDS) established that the original measurement, referring to a visual binary, was never confirmed and that this source should not be regarded as double. Following an information from the referee, we note that while WEI 15 was included in the Index Catalogue of Visual Double Stars (Jeffers et al. 1963), it has been purged from the Washington Double Star Catalog (Mason et al. 2001; Worley \& Douglass 1997).

SAO 161192 is a wide visual binary (48" along $P A=$ $\left.132^{\circ}\right)$. The companion was not included in our diaphragm. We note that this source was reported unresolved also in two previous LO works (Radick \& Lien 1980; Evans \& Edwards 1981), which presumably also referred to the primary only.

\section{Conclusions}

We have reported on lunar occultation observations in the near-infrared of seventeen sources. The results include the discovery of one new triple and eight new binary systems. They also include two stars previously reported as binary by Hipparcos, and for which some discrepancy with our results is apparent. One of them is found to be triple. In the case of two stars which are well-known binaries, one of them being the famous young stellar object GG Tau A, our results provide additional data for the definition of their orbital parameters. Finally, four stars known to be binary could not be detected as such. For one of them, our observation is difficult to reconcile with the parameters of the presumed binary, while a convincing explanation could be found for the other cases.

Acknowledgements. This research has made use of the Simbad database, operated at CDS, Strasbourg (France). In addition to this service, we are glad to acknowledge the fast response of the CDS staff regarding our inquiries about the misidentification of SAO 96753 as a binary star. We are grateful to $\mathrm{H}$. Bulder for providing access to the list of binary stars created and maintained by the International Occultation Timing
Association (IOTA). We are indebted for the support received by two of us, AR and BS, during their observations at the WIRO and SAAO observatories respectively, and we would like to thank in particular R. Howell and I. Glass.

\section{References}

Blow, G. L., Chen, P. C., Edwards, D. A., Evans, D. S., \& Frueh, M. 1982, AJ, 87, 1571

Bonneau, D., Blazit, A., Foy, R., \& Labeyrie, A. 1980, A\&ASS, 42,185

Evans, D. S. 1971, AJ, 76, 1107

Evans, D. S., \& Edwards, D. A. 1981, AJ, 86, 1277

Fabricius, C., \& Makarov, V. V. 2000, A\&A, 356, 141

Fekel, F. C., Montemayor, T. J., Barnes, III T. G., \& Moffett, T. J. 1980, AJ, 85, 490

Guilloteau, S., Dutrey, A., \& Simon, M. 1999, A\&A, 348, 570

Hartkopf, W. I., \& McAlister, H. A. 1984, PASP, 96, 105

Isobe, S., Noguchi, M., Ohtsubo, J., et al. 1992, Pub. Nat. Astron. Obs. Jpn., 2, 459

Jeffers, H. M., van den Bos, W. H., \& Greeby, F. M. 1963, Publ. Lick. Obs., 21

Kaas, A. A. 1999, AJ, 118, 558

Leinert, Ch., Zinnecker, H., Weitzel, N., et al. 1993, A\&A, 278, 129

Mason, B. D., Wycoff, G. L., Hartkopf, W. I., Douglass, G. G., \& Worley, C. E. 2001, AJ, in press

Miura, N., Baba, N., Niino, M., et al. 1992, Pub. Nat. Astron. Obs. Jpn., 2, 561

Nassau, J. J., \& Blanco, V. M. 1957, ApJ, 125, 195

Radick, R., \& Lien, D. 1980, AJ, 85, 1053

Richichi, A., Calamai, G., Leinert, Ch., Stecklum, B., \& Trunkovsky, E. M. 1996a, A\&A, 309, 163

Richichi, A., Baffa, C., Calamai, G., \& Lisi, F. 1996b, AJ, 112, 2786

Richichi, A., Calamai, G., Leinert, Ch., \& Stecklum, B. 1997, A\&A, 322, 202

Richichi, A., Ragland, S., Calamai, G., et al. 1999, A\&A, 350, 491

Richichi, A., Ragland, S., Calamai, G., et al. 2000, A\&A, 361, 594, Paper V

Richichi, A., \& Calamai, G. 2001, in press

Roddier, C., Roddier, F., Northcott, J. E., Graves, J. E., \& Jim, K. 1996, ApJ, 463, 326

Simon, M., Beck, T. L., Greene, T. P., et al. 2000, AJ, 117, 1594

Skiff, B. A. 1999, IAU Inform. Bull. Var. Stars, 4678, 1

Worley, C. E., \& Douglass, G. G. 1997, A\&AS, 125, 523 